\title{
Le travail autonome de l'élève aux Pays-Bas
}

Autonomous learning in the Netherlands

El trabajo autónomo del alumno en Holanda

\section{Sarah Blom et Chantal Weststrate}

\section{(2) OpenEdition}

\section{Journals}

Édition électronique

URL : https://journals.openedition.org/ries/1877

DOI : 10.4000/ries. 1877

ISSN : 2261-4265

\section{Éditeur}

France Education international

Édition imprimée

Date de publication : 1 avril 2002

Pagination : 75-83

ISBN : 2-84520-553-8

ISSN : $1254-4590$

\section{Référence électronique}

Sarah Blom et Chantal Weststrate, « Le travail autonome de l'élève aux Pays-Bas », Revue internationale d'éducation de Sèvres [En ligne], 29 | avril 2002, mis en ligne le 24 novembre 2011, consulté le 05 juillet 2021. URL : http://journals.openedition.org/ries/1877 ; DOI : https://doi.org/10.4000/ries.1877 


\section{Le travail autonome de l'élève aux Pays-Bas}

\section{Sarah Blom et Chantal Weststrate}

Au cours de la dernière décennie, l'élève "soumis "s'est transformé aux Pays-Bas en élève " autonome ». Cette évolution rapide, qui s'étend à tous les niveaux du système éducatif, a des racines plus anciennes. À partir de la fin du XIX ${ }^{\mathrm{e}}$ siècle, une pédagogie naturaliste, soutenue plus tard par la psychologie du développement de l'enfant et par la psychologie différentielle, a mis l'accent sur le développement naturel des capacités innées de l'enfant. Les enseignants ont incorporé ces idées à leur habitus : «suivre» le développement de l'enfant et " accepter », sinon, " organiser les différences entres les élèves » sont devenus des credos importants. Cette volonté de différencier correspondait à une dynamique de différenciation plus étendue dans la société néerlandaise (De Voogd, 1992 ; Blom, 1995). Au niveau du système éducatif, cette tendance à différencier s'est traduite par un enseignement en colonnes laissé à la liberté de choix des parents. De plus, l'ancienne différenciation sociale a survécu dans des filières homogènes hiérarchisées dans le secondaire et en une pédagogie de différenciation interne dans le primaire, ainsi que dans les filières parfois moins homogènes de la première année du secondaire. À l'âge de douze ans, la plupart des élèves sont déjà orientés et casés dans une filière assez fermée (voir tableau p. 76). Dans le premier cycle du secondaire, il existe au moins cinq filières. Dans le deuxième cycle du secondaire, les élèves sont orientés vers l'une des trois filières de l'enseignement général (35\% d'une génération) ou vers l'enseignement professionnel offrant des niveaux et des programmes divers (taux moins stable, d'environ $55 \%$ ).

L'enseignement tertiaire se divise en enseignement universitaire (y sont admis les élèves ayant un bac classique ou moderne) et enseignement professionnel (y sont admis les élèves ayant un bac technologique ou professionnel).

C'est dans ce contexte institutionnel qu'il faut situer l'évolution vers l'apprentissage autonome de l'élève. Nous décrirons d'abord la politique récente des ministres de l'éducation nationale. Celle-ci a visé à introduire une pédagogie nouvelle de l'apprentissage autonome, d'abord dans le primaire, ensuite dans le secondaire, culminant avec la "Maison des études» dans le deuxième cycle de l'enseignement général et technologique. Ensuite nous aborderons la réalisation de cette politique dans un champ éducatif à la fois dynamique, résistant et fatigué. Comment le nouvel encadrement du travail de l'élève est-il reçu par les établissements? Que signifie-t-il pour les élèves ? Nous finirons par indiquer les thèmes de débats, et quelques résultats évaluatifs, encore précoces, mais nullement inattendus. 
Tableau des principales filières de l'éducation secondaire aux Pays-Bas ${ }^{1}$

\begin{tabular}{|c|c|c|c|}
\hline $\begin{array}{c}\text { VWO } \\
\text { Education } \\
\text { pré-universitaire }\end{array}$ & $\begin{array}{c}\text { HAVO } \\
\text { Enseignement général } \\
\text { secondaire supérieur }\end{array}$ & \multicolumn{2}{|c|}{$\begin{array}{c}\text { VMBO } \\
\text { Enseignement secondaire } \\
\text { pré-professionnel }\end{array}$} \\
\hline $\begin{array}{l}\text { Prépare à l'université } \\
\text { Deux formes : } \\
\text { - gymnasium (lycée } \\
\text { classique) } \\
\text { - atheneum (lycée } \\
\text { moderne) } \\
6 \text { années : } 3+3 \\
\text { élèves de } 12 \text { à } 18 \text { ans }\end{array}$ & $\begin{array}{l}\text { Prépare à l'enseignement } \\
\text { professionnel supérieur }\end{array}$ & $\begin{array}{c}\text { MAVO } \\
\text { Enseignement général } \\
\text { secondaire moyen } \\
\text { Prépare à l'enseignement } \\
\text { secondaire professionnel } \\
\text { du } 2^{\text {nd }} \text { cycle } \\
4 \text { années } \\
\text { élèves de } 12 \text { à } 16 \text { ans }\end{array}$ & $\begin{array}{c}\text { VBO } \\
\text { Enseignement } \\
\text { secondaire } \\
\text { professionnel du } 1^{\mathrm{er}} \text { cycle } \\
\text { Acquisition de méthodes } \\
\text { de travail } \\
4 \text { années } \\
\text { élèves de } 12 \text { à } 16 \text { ans }\end{array}$ \\
\hline
\end{tabular}

1. N’ont pas été pris en compte l'enseignement secondaire spécial ni la formation pratique. La loi de 1968 qui est à l'origine de cette organisation prévoit de nombreuses passerelles entre ces différents enseignements, avec un système d'examens et d'équivalences. Il existe également une première année commune appelée classe-pont, qui permet de combler le fossé entre le primaire et le secondaire et de déterminer le type d'école le plus approprié à l'enfant. Sources : The education system in the Netherlands- Eurybase 2001 (Base de données d'Eurydice sur les systèmes éducatifs européens), 2000 ; Laurent Réguer : Le système éducatif des Pays-Bas, Sèvres, CIEP, 1996.

\section{Politique nationale}

L'idée de l'apprentissage autonome n'a pas surgi seulement du mouvement de la pédagogie nouvelle. Tout en existant en marge du système éducatif depuis le début du XXe siècle, elle a depuis les années soixante été développée par des enseignants innovateurs qui y voyaient d'abord une alternative à la pédagogie traditionnelle - le cours magistral en particulier - devenue trop dépersonnalisée pour un enseignement de masse.

Pendant les années soixante-dix, un courant fort dans l'opinion publique s'est opposé à l'intellectualisme (les objectifs uniquement cognitifs) dominant les écoles, ce qui a conduit à modifier les objectifs principaux de l'école primaire. Il s'est agi, dès lors, de promouvoir le développement social, affectif et cognitif. L'activité de l'élève est devenue le centre d'intérêt autour duquel les apprentissages et les matières devaient être articulés. Les élèves du primaire devenant de plus en plus apprenants autonomes, le public eut l'impression que dans le secondaire cette autonomie disparaissait devant une pédagogie traditionnelle. On a alors considéré que le secondaire devait privilégier l'épanouissement des élèves autant que le primaire. Cet épanouissement serait obtenu dans un collège unique (middenschool) où chaque élève atteindrait ses objectifs individuels.

Il faut dire que cette tendance vers une pédagogie "douce » a provoqué une résistance sérieuse, suscitée non seulement par les enseignants et les parents des lycées généraux (craignant une baisse du niveau), mais aussi par les changements économiques. Le système éducatif n'absorbait-il pas une grande partie du budget national sans que le public voit un résultat clair ? Voilà pourquoi, au 
lieu d'accentuer la liberté d'épanouissement des élèves, les politiciens se sont attachés de nouveau aux objectifs intellectuels et à un rendement visible. C'est pourquoi, dans les années quatre-vingt, l'innovation dans l'enseignement secondaire allait porter sur un tronc commun et des objectifs généraux à atteindre. Un programme pour une formation de base (basisvorming) jusqu'à la fin de la scolarité obligatoire à plein temps ( 15 ans) en fut le produit. Cette innovation n’a pourtant pas réussi à apaiser le débat sur les programmes («trop chargés pour les filières pré-professionnelles", "trop simples pour les plus doués ", etc.), sur les structures du système éducatif (le public continuait à identifier la formation de base au collège unique rejeté), sur la pédagogie (trop ciblée sur l'activité de l'élève) et sur les modes d'évaluation.

Mais vers la fin du $\mathrm{XX}^{\mathrm{e}}$ siècle, l'idée de l'apprentissage autonome de l'élève surgit à nouveau, s'associant aux exigences de la société informatique et à l'individualisation progressive. Cette alliance d'idées apparut fructueuse aux yeux des politiciens, moins occupés de l'état de l'éducation nationale que de la réduction des dépenses du secteur public. Dans la perspective d'une introduction générale des TIC, l'élève travaillant de façon autonome représenterait une chance : la réduction du nombre d'enseignants et de classes.

Cet objectif économique a provoqué de vives réactions et une forme de démoralisation des enseignants qui a contribué aux difficultés actuelles de recrutement. Mais, d'une part, en raison du sérieux déficit d'enseignants et, d'autre part, d'une nouvelle définition de compétences à acquérir par l'élève au cours du cycle secondaire, le travail autonome constitue une modalité indispensable des apprentissages scolaires.

Quelle est donc, actuellement, la situation dans l'enseignement néerlandais pour ce qui concerne les innovations visant à renforcer le travail autonome de l'élève?

\section{L’ÉtAT ACTUEL DU TRAVAIL AUTONOME L'élève de 12 ans}

À la sortie de l'école primaire, à 12 ans, l'enfant intègre l'enseignement secondaire. Il est capable d'apprendre et de travailler en petits groupes. À la fin de l'école primaire, l'élève a acquis des connaissances générales sur les PaysBas; il connait les dates principales de l'histoire nationale. Il a des connaissances globales de la géographie des Pays-Bas, se repère sur la carte de l'Europe et connaît la topographie des pays voisins. Dans le domaine des langues, il sait lire des livres de jeunesse, des instructions et des journaux régionaux et il est capable d'écrire des textes de structure simple sans faire trop de fautes. Il se débrouille en anglais dans des situations simples de la vie quotidienne. Il sait calculer (additionner, soustraire, multiplier et diviser) et a fait connaissance 
avec quelques phénomènes de la biologie et des sciences physiques. Au niveau des compétences, l'élève est capable de consulter diverses sources telles que le dictionnaire, une encyclopédie simple ou Internet pour y faire des recherches d'information. Il sait participer à des activités guidées, par exemple dans un domaine concernant sa famille, son quartier, sa ville ou sa région. Il est également capable de parler d'un sujet familier devant la classe ou de présenter un petit travail de recherche.

L'aptitude à apprendre en écoutant seulement l'enseignant est moins développée qu'avant puisque presque tout l'enseignement de l'école primaire est ciblé sur le savoir-faire. Actuellement l'acquisition de connaissances joue un rôle moins important que le savoir-faire.

\section{Le premier cycle du secondaire : la formation de base élargie (basisvorming)}

Dans l'enseignement secondaire les élèves sont regroupés dans des classes plus ou moins homogènes. C'est à dire que chaque classe est constituée d'élèves du même niveau d'apprentissage. Le tronc commun du premier cycle (deux tiers des matières, élèves de douze à quinze ans) est identique pour tous les élèves; la différence entre les niveaux est surtout visible au niveau du rythme auquel ce curriculum est abordé. Les élèves du VWO (enseignement pré-universitaire), achèvent la formation de base normalement en deux ans, ceux du $H A V O$ (enseignement général court) l'achèvent pendant la troisième année, et ceux du VMBO (enseignement secondaire à formation professionnelle) en trois ou même quatre ans. Cela veut dire que les kerndoelen (objectifs principaux) doivent être atteints par tous les élèves entre douze et quinze ans. L'ensemble de ces objectifs vise à développer les compétences et les stratégies d'apprentissage et doit élargir les acquis fondamentaux de l'école primaire. Les objectifs prioritaires sont ciblés sur la communication, les moyens d'expression et l'autonomie de l'élève. Les disciplines enseignées doivent permettre le travail personnel et inviter à la réflexion.

Dès l'introduction de ce curriculum en 1993, une variété d'actions innovatrices dans le domaine de la didactique a été mise en place. Bien que les innovations accordent une assez grande autonomie pédagogique aux établissements scolaires, la basisvorming doit dispenser à tous les élèves de tous les niveaux une formation générale permettant d'une part d'acquérir les connaissances et les savoir-faire fondamentaux et d'autre part de les préparer aux formations offertes après la troisième année de l'enseignement secondaire, donc à la fin de la scolarité obligatoire à plein temps.

Bien que l'importance des cours magistraux ait beaucoup diminué maintenant, cette modalité joue toujours un rôle dans l'enseignement secon- 
daire. Dans le cadre des classes homogènes, il n'est pas étonnant que, dans beaucoup d'établissements scolaires, on trouve encore le professeur devant sa classe, expliquant un sujet abstrait ou compliqué, donnant des instructions globales ou menant des exercices oraux. Dans la majorité des établissements ces cours magistraux représentent environs un quart à un tiers de la totalité des cours du premier cycle. Ces cours sont dispensés à des classes qui ont en moyenne une trentaine d'élèves. L'heure de cours compte 45 ou 50 minutes.

Depuis les années soixante-dix, les moyens électroniques, tels que audiocassettes, vidéos et calculatrices occupent une place considérable dans le quotidien de la classe. Depuis la deuxième moitié des années quatre-vingt-dix, l'enseignement est profondément influencé par l'ordinateur. Les élèves de tous les niveaux se sont maintenant familiarisés avec l'utilisation de logiciels pendant les cours. Dans toutes les matières enseignées, les technologies de l'information et de la communication ont acquis une place effective. Peu à peu le quotidien de la classe change et les élèves s'habituent à une plus grande autonomie dans la réalisation des tâches.

La modification des programmes nationaux des années quatre-vingtdix est à la base de vrais changements. La définition de nouveaux objectifs, aussi bien pour le premier que pour le deuxième cycle, s'est traduite par une didactique innovatrice, paradoxalement renforcée par le manque de professeurs. Ces nouveaux objectifs visent de plus en plus les compétences et les stratégies d'apprentissage. Les cours sont organisés en fonction de grilles planificatrices (studiewijzers) qui guident les élèves à travers le curriculum. La vraie dynamique des innovations éducatives se situe au sein de la classe. Ainsi est-il maintenant courant que les élèves travaillent à deux, soit pour préparer une production commune, soit pour vérifier et corriger les réponses, soit pour discuter d'un sujet ou pour trouver la solution d'un problème posé. L'enseignant joue un rôle beaucoup moins directif qu'avant. Son rôle pendant les cours est surtout celui d'accompagnateur.

L'élève dans le premier cycle est pourtant toujours guidé par l'enseignant. C'est celui-ci qui organise le travail à faire par les élèves, élabore la plupart des plannings et donne les instructions. On peut constater pourtant que l'élève travaille souvent dans une certaine autonomie, une autonomie guidée par l'enseignant. Au moins, l'élève apprend de façon bien plus active qu'avant.

La pédagogie du premier cycle trouve ses racines dans les idées de Kolb (1971) selon lesquelles l'élève doit parcourir le cycle d'apprentissage suivant les étapes suivantes :

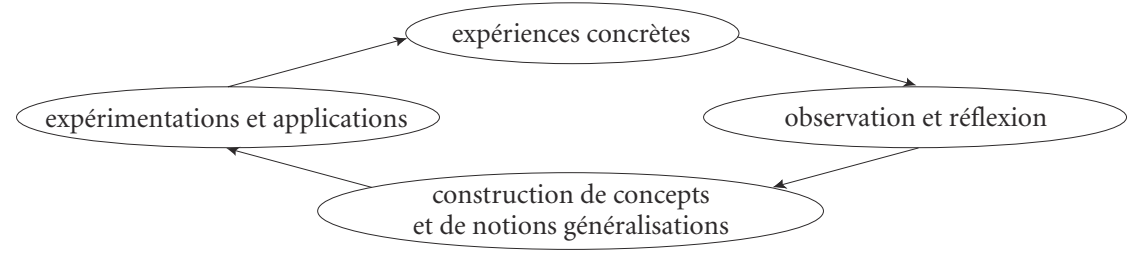


Par exemple, pour la biologie, les élèves de la deuxième classe (treize ans) font un dossier sur la nature dans leur quartier. Ils travaillent souvent à deux et contribuent à part égale à la réalisation du dossier. Ils prennent conscience de leur environnement quotidien, font des observations et les notent. À partir de leurs réflexions, ils construisent des notions et font des généralisations sur la nature dans la ville. Ensuite ils appliquent ce qu'ils ont trouvé et présentent le tout dans un dossier. L'objectif d'un tel travail est surtout de savoir trouver l'information adéquate, de la classer, d'interpréter les données et de savoir présenter ces données. Le travail en équipe des élèves impliquant la responsabilité de leur propre apprentissage est aussi un objectif important pendant ce type d'activité.

Autre exemple d'activité autonome pris dans l'éducation religieuse. En classe, le professeur discute avec les élèves des différentes religions présentes dans le groupe d'élèves. Il y a peut-être des enfants d'origine musulmane, juive ou hindoue. On parle des différences et des ressemblances entre ces religions. Ensuite, les élèves passent aux activités autonomes pour la production d'un dépliant sur la diversité religieuse dans la commune où ils habitent. Pour ce faire, ils situent sur le plan de la ville au moins cinq lieux de culte différents (église, temple, mosquée, synagogue, etc.). Le travail doit être effectué à l'aide de l'annuaire, des pages jaunes et d'autres sources d'information publiques. Ainsi ils peuvent trouver aussi d'autres informations telles que le nombre de croyants par religion, les jours de culte et le nom des prêtres (imam, pasteur, curé, etc.). Enfin, ils classent toutes les informations trouvées et les présentent sous forme d'un petit dépliant illustré.

L'évaluation du travail des élèves a lieu trois fois par an, sous forme de bulletin contenant les notes de l'élève et les appréciations des professeurs. Les notes du bulletin représentent la moyenne des épreuves écrites et orales, et des travaux pratiques. On note de 1 à 10. À la fin de chaque période d'apprentissage, le conseil des professeurs détermine ce qui a été réalisé par chaque élève aussi bien dans le domaine de l'acquisition des connaissances que dans le processus d'apprentissage. Il n'existe pas de réglementation nationale concernant la notation. À la fin du premier cycle, chaque élève fait l'objet d'une évaluation qui conditionne son orientation. Chaque établissement scolaire a la liberté d'organiser son propre système d'évaluation. Ce système d'évaluation est cependant public : il doit être publié dans le guide scolaire ou même sur Internet.

\section{Le deuxième cycle : filières générales et technologiques (Tweede Fase et Studiehuis)}

Avec le bagage acquis pendant la formation de base, l'élève de quinze ans peut entrer dans le deuxième cycle de l'enseignement secondaire, dont les filières générales et technologiques sont appelées "deuxième phase» et "maison des 
études» et durent deux ou trois ans. Cette deuxième phase représente une réforme profonde. Elle est en vigueur depuis 1998. Son objectif central est l'apprentissage autonome de l'élève qui doit assumer une grande responsabilité vis-à-vis de sa propre progression. Cette autonomie accrue doit lui faciliter l'accès à l'enseignement supérieur. Pour atteindre cet objectif d'autonomie, l'élève dispose d'un éventail d'outils, comme ceux acquis pendant le premier cycle.

On pourrait dire que le PTA, la grille d'évaluation et d'examen, sert de fil conducteur à travers le programme de la "deuxième phase ", constituant ainsi le cœur du curriculum.

Qu'est-ce que cela signifie pour l'élève? Tout d'abord l'inconvénient de se trouver confronté à une paperasserie considérable. Pour chaque matière de sa filière personnelle, l'élève réalise un certain nombre de productions écrites autonomes qu'il doit rassembler et conserver dans de nombreux dossiers. Avant de passer les examens scolaires, l'élève doit présenter l'ensemble des dossiers à ses professeurs. Ces dossiers ne sont pas notés par l'enseignant, mais celui-ci doit y apporter la mention "suffisant » afin que l'élève puisse effectivement participer aux examens. Cette contrainte, malheureusement inévitable, est largement compensée par la liberté de l'élève de personnaliser ses dossiers. Il en résulte forcément une différenciation des travaux rendus par les élèves.

Dans le domaine des langues étrangères, l'anglais est obligatoire pour tous les élèves à chaque niveau. Mais, depuis l'introduction de la deuxième phase, l'enseignement du français et de l'allemand a été scindé en deux. Selon sa filière, l'élève suit des classes consacrées à des compétences partielles. Dans la filière des langues seulement, profil «culture et société », l'élève apprend l'ensemble des compétences linguistiques, compréhension orale et écrite, expression orale et écrite et littérature. La plupart des élèves, donc ceux qui ont choisi une des trois autres filières, ne suivent qu'une compétence partielle, soit la compréhension écrite (dans la filière $V W O$ ), soit la compréhension et l'expression orales (dans la filière $H A V O$ ). Les compétences partielles font partie du tronc commun du programme. La raison de cette répartition de compétences entre les deux filières générales (gymnasium, atheneum) et technologiques (HAVO) réside dans les exigences de l'enseignement supérieur. Les diplômes généraux préparent à l'enseignement universitaire et le $H A V O$ aux formations professionnelles supérieures. Les universités demandent aux étudiants de savoir lire des textes scientifiques dans plusieurs langues étrangères, traditionnellement l'anglais, l'allemand et le français. Les formations professionnelles supérieures exigent souvent des stages à l'étranger où une bonne communication orale est élémentaire. Il y a actuellement un débat important à l'intérieur de l'enseignement sur l'efficacité de ces compétences partielles.

Un autre aspect remarquable du curriculum est celui des visites autonomes des élèves au théâtre, au concert, au musée, à des expositions ou au cinéma. Ces visites ont lieu dans le cadre de $C K V$, une nouvelle matière concer- 
nant les compétences culturelles et artistiques de l'élève. Chaque élève reçoit des bons de réduction lui servant de billets d'entrée. Ainsi il assiste au moins à six manifestations culturelles par année scolaire, selon son goût personnel. Il en écrit un compte rendu dans lequel il donne son opinion. Ce système de visites culturelles personnelles, élément nouveau et fort apprécié par les élèves, a été mis en place dès l'introduction de la "deuxième phase ».

L'élève peut également faire preuve de son autonomie par son choix personnalisé d'œuvres littéraires et par la présentation de l'analyse personnelle de ses lectures. Les objectifs de ce type d'activités visent avant tout à stimuler le développement individuel de l'élève. L'acquisition de connaissances culturelles ou historiques est secondaire.

Dernier exemple d'une tâche typique de la "deuxième phase »: le mémoire de fin d'études secondaires. L'élève en choisit librement le sujet, à condition que deux matières du curriculum soient concernées. Ce mémoire comprend le résultat des recherches et études effectuées par l'élève.

Il s'en suit qu'une autonomie considérable de l'élève est requise au niveau du planning, de la production autonome et de la présentation des travaux dans la mesure où tous les objectifs de la deuxième phase sont formulés dans le sens de l'éducation autonome et permanente. En outre, l'examen terminal comporte trois éléments décisifs : les dossiers, y compris le mémoire, les épreuves scolaires et l'examen national. Ces trois éléments permettent d'apprécier l'ensemble des méthodes et des connaissances acquises au cours de la scolarité. La note finale pour chaque matière est calculée sur la moyenne des deux notes attribuées à l'examen scolaire et à l'examen national. L'organisation générale de l'examen est confiée aux établissements scolaires.

\section{Sentiments ambigus}

Il est clair que l'évolution vers un travail plus autonome de l'élève ne peut être jugée qu'en rendant compte de l'évolution du contexte, surtout des idées pédagogiques et des structures scolaires qui se sont développées en concordance. Les bénéfices attendus de cette évolution étant éclaircis ci-dessus, il est temps de regarder de près quelques désavantages.

Premier point négatif : la réduction de l'accès aux filières dites "élitistes ", comme celle du gymnasium, de l'atheneum et du HAVO. C'est que les élèves sont maintenant sélectionnés sur un critère supplémentaire : le travail autonome. En outre, les établissements sont sanctionnés en cas de redoublements. Il n'est donc pas intéressant de garder un élève en retard ou en difficulté. Il sera relégué vers une filière inférieure.

Deuxième point négatif : le manque de choix pour les élèves. En raison des profils et des travaux individuels obligatoires, il ne leur reste que très peu de temps à travailler selon leur volonté. De plus, les élèves de milieux défavo- 
risés éprouvent plus d'angoisse pour les exigences moins explicites et recourent par conséquent au «bachotage»(Blom et Severiens, 2001).

Troisième point, un point de doute, qui concerne la fonction attribuée à l'innovation. Celle-ci devrait améliorer l'insertion des diplômés dans l'enseignement tertiaire. Pourtant, maintenant que les premiers diplômés " autonomes» entrent dans les universités et dans les grandes écoles, celles-ci ont recours à une discipline plus stricte. De plus, ayant besoin d'un flux plus large, elles viennent d'annoncer leur intention de ne pas prendre en compte le profil de l'étudiant.

Enfin, il ne faut pas oublier les enseignants qui se trouvent dans une situation peu enviable. Le manque de professeurs ronge l'énergie de ceux qui restent et qui se sont sentis manipulés par le ministère de l'Éducation dans sa tentative inquiète pour apaiser les controverses sur les innovations. Même ceux qui ont accueilli les innovations de façon enthousiaste considèrent excessive la charge de travail qu'un enseignant néerlandais est tenu de donner : 26 cours par semaine, c'est-à-dire le maximum parmi les pays de l'Union européenne. Cette charge est d'autant plus pénible qu'une grande partie des élèves donne l'impression de fréquenter l'école pour voir les copains et non pas pour écouter le prof.

$\mathrm{Au}$ fond, il est trop tôt pour juger.

\section{BiblographIE}

BLOM S.V. (1995): Formation intellectuelle aux Pays-Bas et en France, Groningen, Wolters-Noordhoff.

BLOM S.V. (1996) : "Fonctionnement de systèmes éducatifs et représentations dominantes du développement intellectuel de l'enfant», article, Genève, Conférence Piaget-Vygotsky.

BLOM S.V., SEVERIENS S. (2001) : "Group differences in self-regulated learning ", article, AERA, Seattle.

KRIJSMAN A., WESTSTRATE Ch. (1997): Le francais et l'allemand au second cycle, Livre de ressources, Thieme, Zutphen.

VOOGDD Ch. De. (1992) : L'histoire des Pays-Bas, Turin, Hatier.

www.min.OCW.nl 